\title{
Cardiac dysfunction from cancer and cancer therapy: new pathways for the prevention of late cardiotoxicity
}

\author{
Lars Michel $^{1} \cdot$ Matthias Totzeck $^{1} \cdot$ Tienush Rassaf $^{1}$
}

Published online: 20 October 2021

(c) The Author(s) 2021

This editorial refers to 'Anthracycline-free tumorelimination in mice leads to functional and molecular cardiac recovery fromcancer-induced alterations in contrast to long-lasting doxorubicin treatmenteffects', by Pietzsch et al.

While the toxic effects of common cancer therapies on the cardiovascular system are increasingly understood mechanistically, the precise direct effects of cancers on cardiovascular diseases remain incompletely resolved $[6,18,24]$. This can in part be related to two major problems: (i) in clinical routine, cancer-related effects can hardly be separated from cancer therapy-induced cardiotoxicity, and (ii) many preclinical studies lack rigorous cancer models and cardiotoxicity is frequently tested in healthy specimens. As cancer itself may pose a major burden to cardiovascular health with significant consequences on cardiovascular outcomes, the understanding of the underlying mechanisms is crucial for a comprehensive understanding of the biology.

Advanced cancer is commonly associated with cardiac atrophy, leading to myocardial structural alterations, cardiac metabolism and myocardial remodelling. A decline in skeletal muscle mass is paralleled by a rarefication of the contractile apparatus in myocardial tissue with decreased gene expression of troponin I, associated with increased fibrosis and a reduced functional capacity, as reflected by decreased fractional shortening and cardiac output [3, 22]. Tumor-associated factors were shown to induce changes in cardiac redox homeostasis resulting in higher levels of reactive oxygen species, reduced mitophagy, and lower hypoxia resistance [9]. Glucose metabolism appears to exhibit a further key element of cancer-induced cardiomyopathy. Low

This comment refers to the article available at https://doi.org/10. 1007/10.1007/s00395-021-00902-7.

Tienush Rassaf

Tienush.Rassaf@uk-essen.de

1 Department of Cardiology and Vascular Medicine, West German Heart and Vascular Center, University Hospital Essen, Hufelandstraße 55, 45147 Essen, Germany insulin plasma levels in cancer cachexia lead to reduced glucose uptake in cardiac tissue and promote cardiac atrophy and subsequent systolic heart failure. In skeletal muscle, an increased insulin resistance is found, and skeletal muscle degradation provides energy substrates through proteolysis that in part promote hepatic gluconeogenesis [3]. In parallel, cardiac triglyceride levels decrease in models for advanced cancers, while CD36 (fatty acid translocase) on cardiomyocytes is upregulated [21]. Systemic effects of cancer on multiple signalling pathways were, furthermore, identified. Exemplarily, cancer alters the growth factor-regulated phosphoinositide 3-kinase (PI3K)-AKT pathway [7] that is also profoundly involved in myocardial signalling with diverse downstream effects [5].

Of note, growing evidence adds another dimension to the diverse coherences of heart and cancer, as cardiovascular disease may also accelerate tumor growth. Preclinical findings indicate that heart failure may promote progression of cancer, as seen in a heart failure mouse model that indicated a correlation between worsening left ventricular function and tumor growth. A similar effect was recapitulated in a transverse aortic constriction mouse model, showing that cardiac hypertrophy/remodelling in the absence of systolic heart failure induced accelerated tumor growth. Several inflammatory factors secreted by the myocardium including periostin and serpinA 3 were proposed to promote tumor growth, as pro-inflammatory biomarkers correlate with newonset cancer in humans $[1,11]$.

Cardiac cachexia negatively impacts morbidity and mortality of patients at risk, and effective therapies have not yet been identified [10]. In cancer patients, cachexia with potential adverse consequences for cardiac integrity affects up to $74 \%$ of cancer patients, with significant consequences on long-term cardiovascular health [3]. Understanding the underlying pathomechanisms and their implications on acute and late cardiac dysfunction is crucial to optimize the management of cardio-oncology patients $[6,15,18,23]$. 
In this issue of Basic Research in Cardiology, Pietzsch and colleagues shed new light on the often neglected impact of the underlying cancer on the recovery after cancerinduced cardiomyopathy [16]. As a novel approach, the authors establish a reversible melanoma mouse model on the basis of the common B16F10 melanoma cell line that was genetically modified via lentiviral transduction of a suicide gene that could induce cell death upon exposure to ganciclovir. With this, the authors were able to observe the effects of cancer on cardiac integrity and its subsequent elimination independently from a specific cancer therapy. To elaborate on therapy-related effects, the authors compared these mice to specimens that were treated with doxorubicin as the prototype of cardiotoxic cancer therapy. Finally, molecular findings were confirmed ex vivo in neonatal rat cardiomyocytes (Fig. 1).

With their in vivo approach, the authors determined that cancer-related cardiomyopathy was fully reversible after therapy-free tumor elimination. Several key metabolic and functional pathways that were shown to deteriorate during advanced cancer including glucose metabolism (GLUT1 and GLUT4 expression, plasma insulin), lipid metabolism (cardiac expression of CD36) and gene expression profiles normalized after a recovery period of $70 \pm 5$ days. Biochemical observations were paralleled by recovered physical strength, a physiological response to angiotensin II exposure and normalized functional parameters. Strikingly, mice that received doxorubicin treatment did not show complete recovery despite tumor elimination. Particularly, mice showed sustained changes in cardiac gene expression patterns that particularly involved circadian rhythm genes, such as Bmal1, Clock, Period1-3, and Cryptochrome 1/2. To determine the effect of this, Bmall was silenced by si-RNA in neonatal rat cardiomyocyte culture, which lead to a reduced mitochondrial membrane potential and increased release of cytochrome $\mathrm{C}$, leading to an increased rate of apoptosis in affected cells. The authors hereby concluded that the observed changes of circadian rhythm genes exhibited significant effects on cardiomyocyte integrity and that its disruption by doxorubicin therapy can promote cardiomyocyte apoptosis, leading to a

- CM size reduction

- Altered cardiac protein homeostasis

- Dysregulated glucose metabolism

- Altered cardiac gene expression

- Cancer metabolites


Fig. 1 Recovery from cancer-induced cardiomyopathy. Scheme of cancer-related cardiomyopathy in the reversible B16F10 transgenic melanoma mouse model and its recovery in the absence and presence of doxorubicin therapy. $B W$ body weight, $C M$ cardiomyocytes, $G C V$ ganciclovir 
persisting disruption of cardiac integrity in contrast to the otherwise fully reversible effects of cancer on the heart.

Pietzsch and colleagues now propose that cancer induces substantial functional, metabolic, and inflammatory modifications, which may be reversible or serve as a potential therapeutic target. This is meticulously assessed for anthracyclines by the authors. Currently, cardiac long-term effects and their recovery from cancer itself lack a clinical demonstration and play a subordinate role in the risk assessment of patients, while therapy-related effects are extensively characterized and included in cardiac risk assessment. Arguably, a mechanistic separation of cancer- and cancer therapy-related effects cannot be easily achieved for all forms of cancer therapies. In a collective of lung cancer patients receiving ICI therapy, more frequent severe immune-related adverse events were found in patients with high tumor burden (odds ratio 8.62) [19], and a comparable observation was demonstrated in a melanoma mouse model, where early cardiotoxicity from ICI therapy in the form of left ventricular dysfunction was only found in tumor-bearing mice [12, 14]. In CAR-T cell therapy, cytokine release syndrome (CRS) often leads to adverse cardiovascular reactions. The severity of CRS is difficult to predict in individual patients, and tumor burden is a main risk factor that is associated with severe CRS [4, 8]. Hence, presence of a tumor and the overall tumor burden will likely impact toxicity in these modern forms of oncological treatment.

Circadian rhythm has been a largely neglected factor in both cardio-oncology and cardiovascular medicine in general $[2,17]$. The authors identify distinct persisting changes in cardiac circadian rhythm pathways only upon exposure to doxorubicin, including downregulation of the core clock protein BMAL1. While this association was already proposed before [2], the authors go further by demonstrating that silencing of Bmall induces elevated apoptosis ex vivo, thereby proving a causative role of circadian rhythm signalling in the development of cancer therapy-related cardiotoxicity. It remains to be said that it is still not fully understood so far to what extent cancer itself influences the degree of cardiotoxicity caused by anthracyclines, since a comparison of treated mice with cancer to a control group of mice without cancer is crucial. The next developments in the field of basic science on the mechanisms of chemotherapy-associated cardiotoxicity can be eagerly awaited here.

Finally, the practical relevance for the clinical work remains, as consequences cannot be drawn based on the basis of the new data from the present study. Decreased expression of GLUT1 and GLUT4 together with lowered insulin levels corroborate the important role of glucose metabolism in maintaining cardiac integrity in cancer patients. It remains elusive whether monitoring of biomarkers, e.g., plasma insulin/c-peptide may serve as a novel risk factor [13]. Pathological findings may, however, provide new therapeutic avenues for medical and non-medical interventions including insulin supplementation as proposed before or exercise therapy, considering its substantial role on glucose metabolism in patients [20,21]. Undoubtedly, the findings show a great potential for future research aiming to gain a broader understanding of the complex mechanisms behind cardiotoxicity from cancer and cancer therapy.

Acknowledgement We acknowledge the use of elements from Servier Medical Art https://smart.servier.com/ Accessed 01 January 2021) for the preparation of the figure.

Funding Open Access funding enabled and organized by Projekt DEAL.

\section{Declarations}

Conflict of interest Matthias Totzeck and Tienush Rassaf report personal fees and other from Edwards, Novartis, personal fees and other from Bristol Myers Squibb, personal fees and other from Bayer, personal fees and other from Daiichi Sankyo, personal fees and other from AstraZeneca, outside the submitted work. Lars Michel declares that there is no conflict of interest.

Open Access This article is licensed under a Creative Commons Attribution 4.0 International License, which permits use, sharing, adaptation, distribution and reproduction in any medium or format, as long as you give appropriate credit to the original author(s) and the source, provide a link to the Creative Commons licence, and indicate if changes were made. The images or other third party material in this article are included in the article's Creative Commons licence, unless indicated otherwise in a credit line to the material. If material is not included in the article's Creative Commons licence and your intended use is not permitted by statutory regulation or exceeds the permitted use, you will need to obtain permission directly from the copyright holder. To view a copy of this licence, visit http://creativecommons.org/licenses/by/4.0/.

\section{References}

1. Avraham S, Abu-Sharki S, Shofti R, Haas T, Korin B, Kalfon R, Friedman T, Shiran A, Saliba W, Shaked Y, Aronheim A (2020) Early cardiac remodeling promotes tumor growth and metastasis. Circulation 142:670-683. https://doi.org/10.1161/circulationaha. 120.046471

2. Ferreira LL, Cervantes M, Froufe HJC, Egas C, Cunha-Oliveira T, Sassone-Corsi P, Oliveira PJ (2020) Doxorubicin persistently rewires cardiac circadian homeostasis in mice. Arch Toxicol 94:257-271. https://doi.org/10.1007/s00204-019-02626-z

3. Fonseca G, Farkas J, Dora E, von Haehling S, Lainscak M (2020) Cancer cachexia and related metabolic dysfunction. Int J Mol Sci. https://doi.org/10.3390/ijms21072321

4. Ghosh AK, Chen DH, Guha A, Mackenzie S, Walker JM, Roddie C (2020) CAR T cell therapy-related cardiovascular outcomes and management: systemic disease or direct cardiotoxicity? JACC CardioOncol 2:97-109. https://doi.org/10.1016/j.jaccao.2020.02. 011

5. Heusch G (2020) Myocardial ischaemia-reperfusion injury and cardioprotection in perspective. Nat Rev Cardiol 17:773-789. https://doi.org/10.1038/s41569-020-0403-y 
6. Heusch G, Rassaf T (2021) Protection from cardiotoxicity of cancer chemotherapy: a novel target for remote ischaemic conditioning? Cardiovasc Res 117:985-986. https://doi.org/10.1093/cvr/ cvaa199

7. Hoxhaj G, Manning BD (2020) The PI3K-AKT network at the interface of oncogenic signalling and cancer metabolism. Nat Rev Cancer 20:74-88. https://doi.org/10.1038/s41568-019-0216-7

8. Larson RC, Maus MV (2021) Recent advances and discoveries in the mechanisms and functions of CAR T cells. Nat Rev Cancer 21:145-161. https://doi.org/10.1038/s41568-020-00323-z

9. Lee DE, Brown JL, Rosa-Caldwell ME, Perry RA, Brown LA, Haynie WS, Washington TA, Wiggs MP, Rajaram N, Greene NP (2021) Cancer-induced cardiac atrophy adversely affects myocardial redox state and mitochondrial oxidative characteristics. JCSM Rapid Commun 4:3-15. https://doi.org/10.1002/rco2.18

10. Lena A, Ebner N, Anker MS (2019) Cardiac cachexia. Eur Heart J Suppl 21:L24-127. https://doi.org/10.1093/eurheartj/suz241

11. Meijers WC, Maglione M, Bakker SJL, Oberhuber R, Kieneker LM, Jong Sd, Haubner BJ, Nagengast WB, Lyon AR, Vegt B, Veldhuisen DJv, Westenbrink BD, Meer P, Silljé HHW, Boer RAd (2018) Heart failure stimulates tumor growth by circulating factors. Circulation 138:678-691. https://doi.org/10.1161/CIRCU LATIONAHA.117.030816

12. Michel L, Helfrich I, Hendgen-Cotta UB, Mincu RI, Korste S, Mrotzek SM, Spomer A, Odersky A, Rischpler C, Herrmann K, Umutlu L, Coman C, Ahrends R, Sickmann A, Löffek S, Livingstone E, Ugurel S, Zimmer L, Gunzer M, Schadendorf D, Totzeck M, Rassaf T (2021) Targeting early stages of cardiotoxicity from anti-PD1 immune checkpoint inhibitor therapy. Eur Heart J. https://doi.org/10.1093/eurheartj/ehab430,10.1093/eurheartj/ ehab430

13. Michel L, Mincu RI, Mahabadi AA, Settelmeier S, Al-Rashid F, Rassaf T, Totzeck M (2020) Troponins and brain natriuretic peptides for the prediction of cardiotoxicity in cancer patients: a meta-analysis. Eur J Heart Fail 22:350-361. https://doi.org/10. 1002/ejhf.1631

14. Michel L, Rassaf T, Totzeck M (2019) Cardiotoxicity from immune checkpoint inhibitors. Int J Cardiol Heart Vasc 25:100420. https://doi.org/10.1016/j.ijcha.2019.100420

15. Mrotzek SM, Lena A, Hadzibegovic S, Ludwig R, Al-Rashid F, Mahabadi AA, Mincu RI, Michel L, Johannsen L, Hinrichs L, Schuler M, Keller U, Anker SD, Landmesser U, Rassaf T, Anker MS, Totzeck M (2021) Assessment of coronary artery disease during hospitalization for cancer treatment. Clin Res Cardiol 110:200-210. https://doi.org/10.1007/s00392-020-01719-5
16. Pietzsch SWK, Thackeray JT, Heimerl M, Schuchardt S, Scherr M, Ricke-Hoch M, Hilfiker-Kleiner D (2021) Anthracycline-free tumor elimination in mice leads to functional and molecular cardiac recovery from cancer-induced alterations in contrast to longlasting doxorubicin treatment effects. Basic Res Cardiol. https:// doi.org/10.1007/s00395-021-00902-7

17. Rabinovich-Nikitin I, Lieberman B, Martino TA, Kirshenbaum LA (2019) Circadian-regulated cell death in cardiovascular diseases. Circulation 139:965-980. https://doi.org/10.1161/CIRCU LATIONAHA.118.036550

18. Rassaf T, Totzeck M, Backs J, Bokemeyer C, Hallek M, HilfikerKleiner D, Hochhaus A, Lüftner D, Müller OJ, Neudorf U, Pfister R, von Haehling S, Lehmann LH, Bauersachs J (2020) Oncocardiology: Consensus Paper of the German Cardiac Society, the German Society for Pediatric Cardiology and Congenital Heart Defects and the German Society for Hematology and Medical Oncology. Clin Res Cardiol 109:1197-1222. https://doi.org/10. 1007/s00392-020-01636-7

19. Sakata Y, Kawamura K, Ichikado K, Shingu N, Yasuda Y, Eguchi Y, Anan K, Hisanaga J, Nitawaki T, Iio M, Sekido Y, Nakano A, Sakagami T (2019) The association between tumor burden and severe immune-related adverse events in non-small cell lung cancer patients responding to immune-checkpoint inhibitor treatment. Lung Cancer 130:159-161. https://doi.org/10.1016/j.lungc an.2019.02.011

20. Scott JM, Nilsen TS, Gupta D, Jones LW (2018) Exercise therapy and cardiovascular toxicity in cancer. Circulation 137:1176-1191. https://doi.org/10.1161/CIRCULATIONAHA.117.024671

21. Thackeray JT, Pietzsch S, Stapel B, Ricke-Hoch M, Lee C-W, Bankstahl JP, Scherr M, Heineke J, Scharf G, Haghikia A, Bengel FM, Hilfiker-Kleiner D (2017) Insulin supplementation attenuates cancer-induced cardiomyopathy and slows tumor disease progression. JCI Insight. https://doi.org/10.1172/jci.insight.93098

22. Tian M, Nishijima Y, Asp ML, Stout MB, Reiser PJ, Belury MA (2010) Cardiac alterations in cancer-induced cachexia in mice. Int J Oncol 37:347-353. https://doi.org/10.3892/ijo_00000683

23. Totzeck M, Mincu RI, Heusch G, Rassaf T (2019) Heart failure from cancer therapy: can we prevent it? ESC Heart Fail 6:856862. https://doi.org/10.1002/ehf2.12493

24. Totzeck M, Schuler M, Stuschke M, Heusch G, Rassaf T (2019) Cardio-oncology - strategies for management of cancer-therapy related cardiovascular disease. Int J Cardiol 280:163-175. https:// doi.org/10.1016/j.ijcard.2019.01.038 